\title{
Derecho de la Libre Competencia
}

Fernando Araya Jasma

Profesor de Derecho Comercial

Universidad Diego Portales

LA DIFICULTAD EN LA PRUEBA DEL DAÑO INDEMNIZABLE EN CASO ALGUNO PUEDE FAVORECER AL AUTOR DEL ILÍCITO ANTICompetitivo. Corte de Apelaciones de Santiago, 7 DE abril de 2016, ROL $\mathrm{N}^{\mathrm{O}} 9.666-2015$

En un interesante desarrollo en materia de aplicación del Derecho de la Libre Competencia por iniciativa privada (private enforcement, en el jerga), la Corte de Apelaciones de Santiago estableció varios criterios que merecen ser destacados por su consistencia con los desarrollos en Derecho europeo de la competencia en este ámbito.

La modificación al DL N 211 introducida por la ley $\mathrm{N}^{\mathrm{O}} 20.945$ de agosto último atribuyó competencia para conocer de las demandas civiles de víctimas contra infractores a las normas de defensa de la libre competencia al propio TDLC, en un procedimiento sumario a ser tramitado una vez ejecutoriada la sentencia infraccional.

Dicho contexto plantea, entre otras, la interrogante sobre qué influencias primarán en las decisiones del nuevo tribunal competente para las indemnizaciones civiles por ilícitos contra la libre competencia. En términos simples, tres alternativas parecen posibles: i) Recoger y extender la evolución jurisprudencial que, sobre esta materia, ha tenido lugar en los tribunales civiles en Chile;

ii) Recepcionar aquí las nociones del Derecho europeo sobre el régimen indemnizatorio como un instrumento adicional para la efectividad de la política de competencia o

iii) Recepcionar aquí la evolución del Derecho estadounidense que en los últimos años ha buscado contener ciertos excesos al que habrían conducido las acciones privadas por su sistema de indemnizaciones equivalentes a tres veces el daño sufrido.

La pregunta parece válida en un asunto que a nivel comparado ofrece soluciones dispares, en cuanto a experiencia, instituciones y a la promoción de reformas. A diferencia del Derecho Infraccional de la Libre Competencia, no existen consensos universales sobre cuál ha de ser el papel de los privados en promover la aplicación efectiva del Derecho de la Libre Competencia. 


\section{LOS HECHOS ANTECEDENTE}

DE LA SENTENCIA EN COMENTO

En un procedimiento iniciado por las demandas acumuladas de cuatro empresas prestadoras del servicio deconversión o terminación de llamadas fijomóvil on-net (empresas de 'celulink'), el TDLC, por sentencia de 15 de octubre de 2009 condenó a TMCH por una de las figuras de abuso de posición dominante de exclusión conocida como estrangulamiento de márgenes. $\mathrm{Al}$ efecto, resolvió el TDLC que TMCH:

"Incurrió en una práctica de discriminación arbitraria de precios, que se tradujo en un estrangulamiento de los márgenes de sus competidores en el mercado de prestación de servicios de terminación de llamadas fijo-móvil on-net, y en una práctica de negativa de venta, con el objeto de traspasar su posición dominante en el mercado de la telefonía móvil al mercado conexo de prestación de servicios de terminación de llamadas fijo-móvil on-net".

El TDLC impuso como medidas contra $\mathrm{TMCH}$

i) una multa de 3.000 UTA, a beneficio fiscal;

ii) la prohibición de cobrar a empresas que ofrecen el servicio de terminación de llamadas fijomóvil on-net, precios arbitrariamente discriminatorios, respecto de los que cobra a sus demás clientes del servicio de telefonía móvil y iii) la obligación de abstenerse en el futuro de conductas similares a la sancionada.

El estrangulamiento de márgenes es un tipo de abuso de exclusión donde una empresa no integrada de forma vertical (en el caso, cualquiera de las empresas de 'celulink') requiere acceder a un insumo indispensable provisto por una empresa integrada verticalmente (en el caso, TMCH) que, en el segmento 'aguas abajo' es, a la vez, competidora de la primera. La empresa integrada verticalmente que, por tanto, es titular de un poder de mercado en el mercado del insumo, explota de modo abusivo su posición de dominio si directa o indirectamente bloquea el acceso al insumo para sus rivales 'aguas abajo'. Una alternativa que tiene a su disposición, es establecer un cobro mayorista suficientemente alto por el insumo y un precio minorista bastante bajo a efectos de estrechar el margen del rival a una cifra insignificante, cero o negativa. El objetivo de exclusión puede llevarlo a cabo con diferentes estrategias tales como una negativa de venta del insumo explícita o implícita, una discriminación arbitraria entre lo que el controlador del insumo cobra a terceros por el acceso al mismo y el precio implícito que 'se cobra a sí mismo', a su unidad verticalmente integrada, por tal acceso o, bien, a través del cobro de precios predatorios 'aguas abajo'. De allí que no sea extraño que una estrategia comercial de este tipo se subsuma a la vez en diferentes categorías jurídicas. En el caso, el insumo en cuestión eran los planes de telefonía móvil (indispensables para la prestación de los servicios de las demandantes), que experimentaron una significativa alza de precios para estos clientes en particular 
(las empresas de 'celulink' demandantes), llevó a la salida del mercado. El siguiente lo que, en último término, junto con esquema describe la estrategia. una decisión del regulador sectorial, los

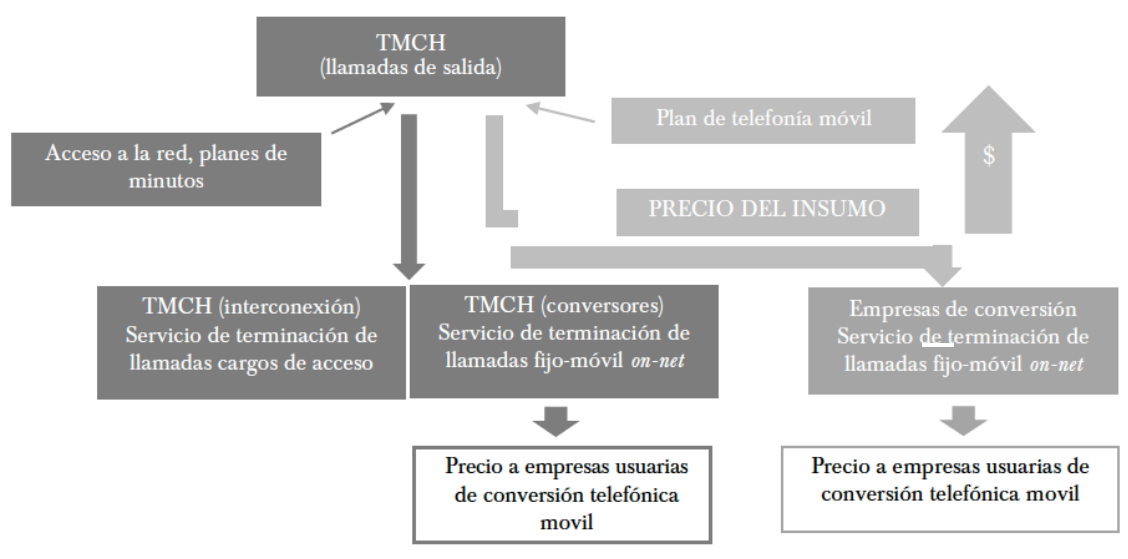

\section{El PROCEDIMIENTO \\ CIVIL INDEMNIZATORIO}

Tratándose de la demanda indemnizatoria presentada por una de las empresas de 'celulink' afectadas, ETCOM', el Vigésimo Juzgado Civil de Santiago desestimó la excepción de ejercicio de actividad ilegal opuesta por la demandada y acogió la demanda imponiendo a TMCH el pago de cien millones de pesos a favor de ETCOM, más reajustes e intereses, sin condenarla en costas².

Por vía de un recurso de casación en la forma de la demandada y de recursos de apelación de la demandada y de la demandante, se elevaron los antecedentes a la Corte de Apelaciones de Santiago la que rechazó la casación

\footnotetext{
${ }^{1}$ La pretensión de ETCOM por el rubro lucro cesante correspondiente a las ganancias que dejó de percibir para el periodo 2008-2014,producto del ilícito de TMCH, ascendía a \$4.219.934.988.

${ }^{2}$ Vigésimo Juzgado Civil de Santiago, 28 de abril de 2015, rol 24.288-2012,ETCOM c. TMCH.
}

en la forma y confirmó la sentencia de primera instancia, con declaración que se eleva a mil doscientos treinta y cinco millones novecientos treinta y cuatro mil doscientos cincuenta y tres pesos el monto indemnizatorio a título de lucro cesante que se ordena que la demandada TMCH pague a la demandante ETCOM, con reajustes e intereses.

Los argumentos de las apelaciones presentadas condujeron a la Corte a resumir las cuestiones controvertidas en las siguientes tres:

i) la alegación de TMCH sobre la ilegalidad de la actividad de la demandante,

ii) la existencia de un daño vinculado causalmente con la infracción, lo que TMCH desconoce $y$

iii) la cuantificación de los daños por el juez de primera instancia, impugnada tanto por $\mathrm{TMCH}$ como por ETCOM ${ }^{3}$.

\footnotetext{
${ }^{3}$ Sentencia comentada (cons. $\left.6^{\circ}\right)$.
} 
En cuanto a la ilegalidad de la actividad de la demandante, para descartar tal defensa, en síntesis, la Corte razona:

i) el TDLC no calificó de ilegal la conducta de la demandante,

ii) la calificación del TDLC no se limitó a lo procedimental sino que se basó además en el fondo de la conducta de ETCOM,

iii) lo establecido por el TDLC sobre el particular resulta "irredargüible e inamovible",

iv) los cargos originales del regulador en contra de ETCOM fueron dejados sin efecto por haber sido formulados con vulneración al principio de tipicidad que rige el ejercicio del ius puniendi estatal ${ }^{4}$.

En cuanto a la existencia de un daño vinculado causalmente con la infracción, la la orden de suspensión de actividades impuesta por el regulador sectorial.

"En este período resulta indubitada la pérdida de la demandante y será el que esta Corte considerará al efecto. No es dable considerar el saldo del tiempo pretendido, desde que, sin duda, resulta que la actora no prestó los servicios de que se trata por causas ajenas a la [...]conducta de su contraria; la suspensión de sus actividades obedeció a la imposición de la Subsecretaría de Transporte y Telecomunicaciones, entidad que, si bien levantó el cargo y, consecuentemente la paralización de actividades de la demandante, no anuló lo obrado en este último aspecto de modo que no pueden entenderse retrotraídas las cosas al estado anterior al cese o interrupción de funciones de ETCOM"

Por último, en cuanto a la cuantificación de los daños, la Corte entre los informes técnicos aportados por la demandante y la demandada, respectivamente, otorga preferencia al aportado por la demandante.

"Por consiguiente, aparece mejor informado, más imparcial, verídico y realista el proporcionado por la actora, del que estos sentenciadores extraerán la pérdida razonable probable de la demandante, aunque siempre en un escenario de razonabilidad y probabilidad, realizando una

\footnotetext{
${ }^{4}$ Sentencia comentada (cons. $9^{\circ}-12^{\circ}$ ).
}

5 Sentencia comentada (cons. $\left.13^{\circ}\right)$.

\footnotetext{
${ }^{6}$ Sentencia comentada (cons. $\left.17^{\circ}\right)$.
} 
regulación, en fin, aproximada, suponiendo el curso normal de la actividad mercantil de la afectada y considerando, además, la dificultad en la prueba de lo que se trata, dificultad que, en caso alguno, puede favorecer al autor del ilícito competitivo"7.

Es hacia final del considerando anterior, precisamente, que se contiene uno de los criterios de mayor interés que se rescata de esta sentencia y sobre el cual volveremos en la siguiente sección al coincidir con orientaciones del Derecho europeo orientadas a alivianar a la víctima la carga probatoria del quantum indemnizatorio. Procediendo derechamente a la cuantificación, la Corte se basa en la proyección del incremento en las ventas de minutos por parte de ETCOM para el año 2008, a partir de los resultados de los años 2006 y 2007, considerando un margen de ganancia menos los gastos de inversión y data center proyectados, lo que arroja una determinada utilidad para el año 2008. Dicha utilidad proyectada para el año 2008, según el informe técnico acompañado por la actora, ascendente a mil doscientos treinta y cinco millones novecientos treinta y cuatro mil doscientos cincuenta y tres pesos corresponde, en definitiva, a la cuantificación del daño indemnizable por la Corte ${ }^{8}$. Del periodo que había originalmente concebido como vinculado causalmente con la infracción (julio 2007-30 de noviembre de 2008) excluye todo el 2007 y considera el 2008 íntegro, lo que explica así:

\footnotetext{
${ }^{7}$ Sentencia comentada (cons. $\left.18^{\circ}\right)$.

${ }^{8}$ Sentencia comentada (cons. $19^{\circ}$ y res. II).
}

"Habida cuenta que se trata de una regulación estimativa y probable y se excluye el año 2007, del que no se cuenta con antecedentes suficientes para precisar la afectación que le produjo a la demandante el alza de precios a contar del mes de abril de ese año" .

Por último, sobre la cuantificación de los daños y la excepción de exposición imprudente al riesgo, la Corte la desestima, declarando:

"Este Tribunal no divisa medida alguna quelademandante pudiera haber adoptado para evitar las consecuencias del alza de precios de que fue víctima y ya se razonó que su conducta no fue ilícita"10.

\section{Criterios a destacar}

A LA LUZ DE LOS DESARROLLOS Del Derecho Europeo

\section{(a) La función prejudicial de la cosa juzgada de la sentencia del TDLC}

La parte considerativa de la sentencia en comento se inicia con una interpretación del alcance del art. 30 del DL N ${ }^{\circ}$ 211. En concepción de la Corte, la disposición otorga, a partir de la sentencia del TDLC, un doble beneficio al titular de la acción indemnizatoria:

i) un procedimiento más breve
y concentrado y
ii) la función positiva o prejudicial
delacosajuzgada, esto es, el efec-

${ }^{9}$ Sentencia comentada (cons. $\left.19^{\circ}\right)$.
${ }^{10}$ Sentencia comentada $\left(\right.$ cons. $\left.20^{\circ}\right)$. 
to de una resolución firme y ejecutoriada queimplica quelamisma servirá de base a ulteriores decisiones de los tribunales ${ }^{11}$.

En criterio de la Corte, los alcances de dichos beneficios no se extienden a:

i) ilícitos anticompetitivos 'sólo de riesgo', sino que debe tratarse de ilícitos anticompetitivos 'concretos', distinción donde la Corte pareciera confundir el daño anticompetitivo con el daño indemnizable ${ }^{12}$; como tampoco se extienden a

ii) situaciones en que a pesar de la existencia de una sentencia condenatoria del TDLC, no sean demostrados los requisitos de la responsabilidad civil extracontractual; ni tampoco a iii) daños ajenos al injusto anticompetitivo $^{13}$.

De la aplicación de esta disposición resulta:

"La consideración imperativa por parte del tribunal civil se limita a las conductas, hechos y calificación jurídica, es decir, del ilícito anticompetitivo establecido en el fallo del [TDLC], debiendo, por consiguiente, demostrarse las restantes exigencias de la responsabilidad extracontractual de la que aquí se trata, esto es, el resultado dañoso y el nexo causal [...], en consecuencia, no le corresponde al sentenciador civil abocarse a la existencia

\footnotetext{
${ }^{11}$ Sentencia comentada (cons. $8^{\circ}$ ).

${ }^{12}$ Araya (2005).

${ }^{13}$ Sentencia comentada (cons. $8^{\circ}$ ).
}

del ilícito propiamente tal, mediante la fijación de la conducta exigida y la adecuación o no de la conducta del rival anticompetitivo a esa exigencia legal" ${ }^{14}$.

La disposición referida y su constante interpretación por los tribunales de justicia que han concebido la sentencia condenatoria del TDLC como base de una verdadera presunción de derecho de la culpa de la demandada es consistente con los desarrollos del Derecho europeo orientados por los principios de efectividad de la política de competencia y equivalencia entre países miembros que, en esta materia, se orienta hacia el establecimiento de responsabilidades civiles sin culpa una vez establecido el ilícito anticompetitivo o presunciones de culpa a partir de dicho establecimiento ${ }^{15}$. La directiva europea en la materia no excluye que los ordenamientos nacionales exijan una imputación subjetiva como elemento, pero tal exigencia está subordinada al cumplimiento de los principios referidos:

"Todas las normas nacionales que regulan el ejercicio del derecho a resarcimiento por los daños y perjuicios ocasionados por una infracción de los artículos 101 o 102 del TFUE, incluidas las relativas a aspectos no abordados en la presente Directiva, como el concepto de relación causal entre la infracción y los daños y perjuicios, han de observar los principios de efectividad y equivalencia. Ello quiere decir

\footnotetext{
${ }^{14}$ Sentencia comentada (cons. $\left.9^{\circ}\right)$.

${ }^{15}$ OECD (2015), pp. 12-14.
} 
que no se deben formular o aplicar de manera que en la práctica resulte imposible o excesivamente difícil el ejercicio del derecho a resarcimiento garantizado por el TFUE, o de modo menos favorable que las aplicables a acciones nacionales similares. Cuando un Estado miembro establezca en su Derecho nacional otras condiciones para el resarcimiento, tales como la imputabilidad, la adecuación o la culpabilidad, ha de poder mantener dichas condiciones en la medida en que se ajusten a la jurisprudencia del Tribunal de Justicia, a los principios de efectividad y equivalencia, y a la presente Directiva" ${ }^{\prime 6}$.

Sin embargo, un cambio en la redacción del art. 30 del DL No 211 introducido con la reforma de la ley $\mathrm{N}^{\circ} 20.945$ resulta llamativo según se consignará en la última sección de este comentario.

\section{(b) El lucro cesante como rubro indemnizable en este ámbito}

Con una oportuna cita al Tratado de Responsabilidad Extracontractual de Enrique Barros, utilizada como base conceptual sobre el lucro cesante, la Corte concluye que, en el caso, este está constituido por:

"La valorización de los minutos que la actora dejó de transar de no mediar el ilícito anticompetitivo, dentro de una proyección razonable del curso de su activi-

${ }^{16}$ Directiva UE (2014), (cons. $\left.11^{\circ}\right)$. dad en el mercado relevante de que se trata, es decir, el de terminación de llamadas fijo-móvil on-net, conexo al mercado de servicios de telefonía móvil, según lo establecido en la sentencia pronunciada por el [TDLC]"17.

La CS ya había establecido que, conceptualmente, el lucro cesante, incluso el lucro cesante futuro, es un rubro reparable en este ámbito, pero en la medida que sea probado ${ }^{18}$.

$\mathrm{Al}$ definir los rubros indemnizables, la directiva europea en la materia establece el principio de la reparación integral o resarcimiento pleno, incluyendo el daño emergente, el lucro cesante o pérdida de beneficios y los intereses, pero que, sin perjuicio de la indemnización por pérdida de oportunidades, un resarcimiento pleno en virtud de tal directiva no debe conducir a un exceso de resarcimiento, ya sea mediante daños punitivos, múltiples o de otro tipo ${ }^{19}$.

Es evidente que la posibilidad de reparar el lucro cesante sería una mera declaración de principios si no fuera acompañada de mecanismos para la alivianar a la víctima la carga probatoria sobre el mismo.

\section{(c) Aligeramiento del estándar de prueba sobre la existencia y cuantificación del daño}

No puede soslayarse en este punto la clara cita de Enrique Barros que la Corte hace suya:

\footnotetext{
${ }^{17}$ Sentencia comentada (cons. $15^{\circ}$ ).

${ }^{18}$ CS 25.07.2013, rol No 1339-2012, Philip Morris Chile con Compañía Chilena de Taba$\cos$ S.A.

${ }^{19}$ Directiva UE (2014), (cons. $\left.12^{\circ}, 13^{\circ}\right)$.
} 
"La determinación del lucro cesante considera un grado razonable de probabilidad en la percepción de los ingresos futuros, y obedece a una proyección del curso normal de los acontecimientos, atendidas las circunstancias particulares de la víctima. Deben proporcionarse antecedentes más o menos ciertos que permitan determinar una ganancia probable. La certidumbre del daño, que la doctrina suele señalar como condición de su reparabilidad, debe ser calificada en el caso del lucro cesante, pues rara vez habrá certeza de que el provecho se habría efectivamente producido. Exige aplicar un cálculo probabilístico de su efectiva ocurrencia. El lucro cesante será determinado usualmente por medio de presunciones e informes periciales" 20 .

A su turno, la Corte contribuye con numerosos pasajes que reiteran las nociones de probabilidad, razonabilidad, curso normal de la actividad comercial, relevando las inherentes dificultades probatorias en este aspecto, destacándose los siguientes:

"La pérdida razonable probable de la demandante, aunque siempre [estimada] en un escenario de razonabilidad y probabilidad, realizando una regulación, en fin, aproximada, suponiendo el curso normal de la actividad mercantil de la afectada" ${ }^{21}$.

\footnotetext{
${ }^{20}$ Sentencia comentada (cons. $15^{\circ}$ ).

${ }^{21}$ Sentencia comentada (cons. $18^{\circ}$ ).
}

"Habida cuenta que se trata de una regulación estimativa y probable" 22 .

"Considerando, además, la dificultad en la prueba de lo que se trata, dificultad que, en caso alguno, puede favorecer al autor del ilícito competitivo"23.

El cálculo de los daños indemnizables puede llegar a ser una tarea muy compleja, entre otras razones, porque los datos y la información muchas veces se encontrará en manos del demandado. La pluralidad de metodologías para la cuantificación puede concluir en divergencias sobre los montos indemnizatorios. Varias jurisdicciones se han hecho cargo de estas dificultades otorgando a los jueces atribuciones más amplias de estimación, liberando a las víctimas de una prueba exacta, estableciendo presunciones de daño y presunciones de montos de daño o derechamente, la posibilidad de obtener la asistencia de las autoridades de competencia en la cuantificación del daño indemniza $b^{24}$. La directiva europea en la materia recoge algunas de estas estrategias:

\section{"Artículo 17 Cuantificación del perjuicio}

1. Los Estados miembros velarán por que ni la carga de la prueba ni los estándares de prueba necesarios para la cuantificación del perjuicio hagan prácticamente imposible o excesivamente difícil el ejercicio del de-

\footnotetext{
${ }^{22}$ Sentencia comentada (cons. $19^{\circ}$ ).

${ }^{23}$ Sentencia comentada (cons. $18^{\circ}$ ).

${ }^{24}$ OECD (2015), pp. 15-17.
} 
recho al resarcimiento de daños y perjuicios. Los Estados miembros velarán por que los órganos jurisdiccionales nacionales estén facultados, con arreglo a los procedimientos nacionales, para estimar el importe de los daños y perjuicios si se acreditara que el demandante sufrió daños y perjuicios pero resultara prácticamente imposible o excesivamente difícil cuantificar con precisión los daños y perjuicios sufridos sobre la base de las pruebas disponibles.

2. Se presumirá que las infracciones de cárteles causan daños y perjuicios. $\mathrm{Al}$ infractor le asistirá el derecho a rebatir esa presunción.

3. Los Estados miembros velarán por que, en los procedimientos relativos a reclamaciones de daños y perjuicios, una autoridad nacional de la competencia pueda ofrecer, previa petición de un órgano jurisdiccional nacional, asesoramiento a este en el tema de la determinación de la cuantía de los daños y perjuicios, si dicha autoridad nacional de la competencia considera adecuado tal asesoramiento.”

De este modo, en no menos de tres aspectos, la sentencia en comento se vincula con los mejores desarrollos orientados a hacer realidad la reparación de las víctimas de los ilícitos contra la libre competencia, a complementar la iniciativa pública en la aplicación de la ley de competencia con la iniciativa privada y, en último término, a refor- zar la efectividad de la política de competencia.

Queda por ver si la CS mantendrá la decisión de la Corte de Apelaciones o si, por el contrario, encontrará en ella vicios que la conduzcan a casar la sentencia y dictar la consecuente sentencia de reemplazo.

\section{Algunas interrogantes}

A LA LUZ DE LA Reforma EN Chile

Queda por ver también, hacia el futuro, cuál será la aproximación a estos temas que tendrán los jueces expertos del TDLC que hasta ahora han conocido solo de la parte infraccional de los ilícitos contra la libre competencia y no del capítulo indemnizatorio de las víctimas.

i) ¿ Verán el tema indemnizatorio sólo con el fin compensatorio o concebirán las indemnizaciones en su doble función compensatoria y disuasoria?

ii) ¿Cómo influirá en la determinación del monto de las multas infraccionales el conocimiento de su capacidad de influir en la determinación del monto indemnizatorio?

iii) ¿Qué estándares de control ejercerán sobre los informes técnicos presentados por las partes sobre cuantificación de daños?

iv) ¿Recurrirán a la FNE en su papel de informante técnico en los procedimientos indemnizatorios?

v) ¿Qué tan estricto o ligero será el estándar de prueba exigido a las partes sobre la existencia 
de los daños, el nexo causal y la cuantificación de los daños? Cabe preguntarse, por último, hacia el pasado, si lo que se modificó en materia de daños por infracciones a la libre competencia con la ley $\mathrm{N}^{\circ} 20.945$ es todo lo bueno que pudo ser.

i) La modificación de la redacción desde la antigua

“...fundará su fallo en las conductas, hechos y calificación jurídica de los mismos, establecidos en la sentencia del [TDLC]...”

a la nueva

“...fundará su fallo en los hechos establecidos en su sentencia que sirvan de antecedentes a la demanda..."

apartaría los desarrollos en Chile de las orientaciones del derecho europeo, si llega a desprenderse que la interpretación aquí es que se ha pasado de un régimen de culpa presumida de derecho a un régimen de culpa probada. Es de esperar que la historia fidedigna del establecimiento de la ley resulte aclaratoria sobre este cambio de redacción.

ii) El infractor por un abuso de posición dominante está, en principio, igualmente expuesto a las demandas indemnizatorias que los miembros de un acuerdo colusorio. Una alternativa pudo optar por profundizar en este ámbito la asimetría entre el tratamiento contra las colusiones y el tratamiento contra los abusos. iii) El primer auto-delator de una colusión está, en principio, expuesto a las demandas indemnizatorias de las víctimas de la colusión, por la totalidad del daño generado por el acuerdo colusorio, al igual que los demás miembros del cartel.

Por las dos razones anteriores, nuestra posición en este punto era llevar al conocimiento del TDLC solo las indemnizaciones por casos de colusión, excluyendo de dicho tratamiento al primer autodelator. Retomar la idea de una responsabilidad simplemente conjunta o mancomunada para el primer autodelator pudo ser otra alternativa a considerar. Durante la tramitación del proyecto de ley que concluyó en la ley $\mathrm{N}^{\mathrm{o}} 20.945$, en el otro extremo se encontraba la posición de CONADECUS que introdujo esta discusión sobre los daños no contemplada en el proyecto original del Ejecutivo, pero con la muy discutible propuesta de condicionar el otorgamiento de los beneficios de la delación compensada al pago u obligación de pago de las indemnizaciones a las víctimas.

iv) Si se hubiese querido brindar un papel más importante a la iniciativa privada en la aplicación efectiva de la ley, quizás se pudo contemplar que en el caso de procedimientos contenciosos ante el TDLC iniciados por demandas de particulares, las demandas civiles indemni- 
zatorias podrían ser tramitadas por cuerda separada sin necesidad de esperar la sentencia ejecutoriada en lo infraccional.

v) Finalmente, han quedado al margen de la legislación y entregadas al desarrollo de la experiencia de aplicación temas tales como el equilibrio entre el acceso a evidencia vs. la protección de los incentivos a la autodenuncia, el acceso amplio a evidencia en poder de la contraparte, la defensa del passing-on y la legitimación activa del comprador indirecto, entre otros.

\section{Bibliografía}

Araya Jasma, Fernando (2005). “Daño anticompetitivo y daño indemnizable: un ensayo de confrontación". Revista Chilena de Derecho Privado. $\mathrm{N}^{\mathrm{O}}$ 4. Santiago.

BANFi del Río, Cristián (2013). "La responsabilidad civil como forma de aplicación privada del derecho de la competencia”. Revista Chilena de Derecho Privado. No 21. Santiago.

Directiva UE (2014) 2014/104/UE del Parlamento Europeo y del Consejo de 26.11.2014 relativa a las acciones por daños por infracciones al Derecho de la Competencia. Disponible en http://eur-lex.europa.eu/legalcontent/ES/TXT/PDF/?uri=CEL EX:32014L0104\&from=EN [Fecha de consulta: 1 de agosto de 2016].

Fuchs Nissim, Andrés y Álvaro Vives Martens (2015). "Dos problemas en torno a la responsabilidad civil derivada de ilícitos anticompetitivos", en Álvaro Vidal Olivares, Gonzalo Severin Fuster y Claudia Mejías Alonso (eds.) Estudios de Derecho Civil X. Santiago: ThomsonReuters

Lewin, Nicolás (2011). "Indemnización de perjuicios por atentados a la libre competencia: el daño anticompetitivo, su relación con el daño civil y la determinación de los perjuicios". Anales de Derecho UC: Temas de libre competencia. $\mathrm{N}^{\mathrm{O}}$ 6. Santiago.

OECD (2015), "Relationship between Public and Private Antitrust Enforcement. Note by the Secretariat" DAF/COMP/WP3(2015)14. Paris. Disponible en http://www.oecd.org/ officialdocuments/publicdisplaydoc umentpdf/?cote $=\mathrm{DAF} / \mathrm{COMP} / \mathrm{WP}$ 3 (2015) $14 \&$ doclanguage $=$ en $[$ Fecha de consulta: 1 de agosto de 2016]. 